\title{
On the sensitivity of the POD technique for a parameterized quasi-nonlinear parabolic equation
}

\author{
Nissrine Akkari ${ }^{1,2^{*}}$, Aziz Hamdouni ${ }^{{ }^{*}}$, Erwan Liberge ${ }^{1}$ and Mustapha Jazar ${ }^{2}$
}

\author{
*Correspondence: \\ nissrine.akkari@univ-Ir.fr; \\ aziz.hamdouni@univ-Ir.fr \\ 1 University of La Rochelle, LaSIE - \\ Laboratory of the Engineering \\ Sciences for the Environment, \\ Avenue Michel Crépeau, 17042 La \\ Rochelle Cedex 1, France \\ ${ }^{2}$ Lebanese University, LaMA - \\ Laboratory of Mathematics and \\ Applications, B.P 826 Tripoli, \\ Lebanon
}

\begin{abstract}
Background: In what follows, we consider the Proper Orthogonal Decomposition (POD) technique of model order reduction, for a parameterized quasi-nonlinear parabolic equation.
\end{abstract}

Methods: A POD basis associated with a set of reference values of the characteristic parameters is considered. From this basis, a parametric reduced order model (ROM) projecting the initial equation is constructed.

Results: A mathematical a priori estimate of the parametric squared $L^{2}$-error induced by this projection is developed. This later estimate is based on both, the parametric behavior of the squared $L^{2}$-ROM-error thanks to the resolution of a Ricatti differential inequality in the parametric ROM-error, and the convergence rate of the parametric ROM to the full problem, via the augmentation of the basis dimension. Indeed, under restrictive conditions on the solutions regularity of such equations, we are able to precise the slope of the logarithm of the squared $L^{2}$-norm of the ROM error, as a function of the logarithm of the basis modes number.

Numerical experiments of our theoretical estimate, are presented for the 2D Navier-Stokes equations in the case of an unsteady and incompressible fluid flow in a channel around a circular cylinder.

Conclusion: A mathematical a priori estimate of the parametric squared $L^{2}$-error induced by the model reduction by POD is developped for a parameterized quasi-nonlinear parabolic equation. This estimate is obtained thanks to the resolution of a Ricatti differential inequality.

Keywords: Quasi-nonlinear equations; ROM; POD; Parametric evolution; Sensitivity; A priori error bound; Burgers equation; 2D Navier-Stokes equations

\section{Background}

\section{Statement of the problem}

High-dimensional Partial Differential Equations (PDE) intervene widely in applications of the field of Mechanics (Fluid Mechanics, Solid Mechanics, etc...). The simulation cost of such equations is in general very high. Model reduction techniques are a very good solution for such problems: They enable an approximation of these equations in subspaces of

() 2014 Akkari et al.; licensee Springer. This is an Open Access article distributed under the terms of the Creative Commons 
small dimension. These are applied for Fluid-Structure Interaction problems [1-7], stability study [8], shape optimization problems $[9,10]$, optimal control problems $[11,12]$ etc....

In general, the reduction spaces are obtained from the knowledge of a solution flow. Then, one of the challenges for model reduction, is to enable a good prediction of the solutions behavior in parametric evolution problems: We cite the Greedy algorithm, that enriches a reduction subspace computed for a particular set of parametric values, by evaluating the errors obtained a posteriori by the reduction of the full numerical solutions (obtained for example by finite element dicretisations of the complete model problem) associated with other parametric values. Obviously, this algorithm needs to be accelerated in a way to enable an estimate of the new parameters' values for which the reduced order model (ROM) error is maximal and the computation of a new reduction basis is becoming crucial. At this step only, a complete resolution of the model equations is done for the new parameters' values chosen by the algorithm. Two important questions can be asked:

What is the parametric confidence region of a given reduced order model?

How can we improve the performance of a reduced order model when parameters are variying significantly?

Several techniques of model reduction exist to build a good candidate within the parametric ROMs:

We consider first the reduced basis (RB) method. It is based on showing a given parametric solution as a finite linear combination of solutions associated respectively with particular parameters values. More precisely, we cite the work of Maday et al. [13,14] who developed, for a parameterized elliptic PDE which is symmetric and coercive, an a priori convergence theory of a parametric RB approximation to the parametric full finite element solution. The RB is constructed with association to a sample subset of parametric values which are logarithmically distributed: this allows an a priori convergence of the Greedy algorithm [15].

They were interested also, in showing an a posteriori bound of the squared parametric $L^{2}$-ROM-RB error, of which computation at each parameter value is less expensive than the parametric ROM-error itself as it is usually done in the original Greedy algorithm. The a posteriori character of this error bound is due to its dependency on the squared residual norm of the parametric ROM. We precise also, that the later error bound is obtained by applying classical energetic methods to the discrete equation in the difference between the full numerical solution and the reduced one, so one can bound the ROM-error by the already precised a posteriori residual norm. This technique was applied in the context of offline-online procedures, in the following cases: parameterized linear elliptic PDE which is coercive and not symmetric [16]; nonaffine linear elliptic and parabolic equations and nonlinear elliptic and parabolic equations [17]; parameterized linear parabolic equation with a nonaffine source term [18]; the model Burgers equation [19,20].

A very adaptive technique, for building a parametric ROM is the Proper Generalized Decomposition (PGD) method. It is based on building an approximation of the initial PDE as a finite combination of functions of separate variables, including not only the space and time variables, but also all eventual parameters that could be associated with the initial equations. These functions and their coefficients in the later expression are obtained by an iterated algorithm which minimizes the error with respect to the initial problem. This method was introduced by P. Ladevèze in the LATIN method [21,22], where he started by 
a space-time separation. Then, it was generalised by Chinesta et al. for multidimensional problems [23-28].

We are discussing in this paper, the parametric sensitivity of a reduced order model by the Proper Orthogonal Decomposition (ROM-POD). A detailed literature on this method can be found in $[29,30]$.

It is still an important issue, to determine the confidence interval of a ROM-POD for problems involving parametric evolution. There were few works in this context:

Amsallem et al. [31-33], build a geometric interpolation algorithm of ROM-PODs at different parametric values, in the case of parameterized linear structural dynamics problems. The idea is to interpolate various reduced order, symmetric and positive definite matrices of the complete problem, corresponding each one to a set of characteristic parameters. This interpolation is done in the tangent space to the manifold of symmetric positive definite matrices. Numerical experiments show that the obtained reduced order model, is efficient to represent dynamics asssociated with other parametric sets than the reference ones.

There are also techniques based on an a posteriori indicator of the POD modes number we need to add, in order to decrease the ROM-error, when the parameters are varying. Volkwein et al. [34-37] were interested in such estimates for solving linear quadratic elliptic and parabolic optimal control problems. Therefore, they developed an a posteriori estimator of the error between the reduced optimal solution and the optimal one. Also, this estimator is based on the residual term we add when solving the reduced adjoint equation. The reduced optimal solution is good when a tolerated error is not trespassed, otherwise we increase the dimension of the reduced optimal control problem.

Besides all these works, we are interested in developing an a priori bound of the squared parametric $L^{2}$-ROM-POD error in the case of a parameterized quasi-nonlinear parabolic equation. More precisely, our main issue is to give a mathematical criteria in order to determine a parametric confidence region for a reference POD basis. It appears also the control problem of the dimension of the associated parametric ROM-POD, so we can improve its performance if the parameters are varying considerably with respect to the reference ones.

This type of result will enable us not only to predict the validity extent of a reference reduced order model of fixed dimension with respect to parametric variation, but also to do an enrichement step of the ROM-dimension when necessary, thanks to an a priori indicator of the number of POD modes we need to add. So that, on the one hand the parametric ROM-error is not maximal when the parameters are varying considerably outside the confidence region determined already, and on the other hand the reduction concept is still available.

The result of this paper is an improvement of the one we developed in $[38,39]$. Where we showed an a priori estimate of the parametric squared $L^{2}$-error induced after the model reduction of parameterized semi-discrete quasi-linear parabolic problems, by a reference POD basis associated with a reference solution for a base parameter value. Indeed, the resolution of a Ricatti differential inequality gives sharper a priori upper bounds compared to the ones we developped in [38,39], where we used the Gronwall lemma to solve a first order differential inequality in the parametric ROM-error. Moreover, we present an improvement of this type of results by considering an enriched POD basis associated with a reference solution and its parametric derivative at the same parameter value. 
More precisely, the mathematical formulation of the problem is given as follows:

\section{Methods}

\section{Mathematical formulation of the problem}

Let us consider a general parameterized quasi-nonlinear parabolic PDE: We denote $X=$ $\left[L^{2}(\Omega)\right]^{d}, V=\left[H^{1}(\Omega)\right]^{d} . \Omega$ is a bounded open set, connected and lipschitz of $\mathbb{R}^{d}$, where $d=1$ or 2 . This equation is given by its weak formulation as follows:

$$
\left\{\begin{array}{l}
\frac{d}{d t}\left(u_{\lambda}(t), v\right)_{X}+B\left(u_{\lambda}(t), u_{\lambda}(t), v\right)+a\left(u_{\lambda}(t), v ; \lambda\right) \\
=S\left(u_{\lambda}(t), v\right)+l_{t}(v) \quad \forall v \in V \\
\left(u_{\lambda}(0), v\right)_{X}=\left(u_{\lambda}^{0}, v\right)_{X} \quad \forall v \in V
\end{array},\right.
$$

where $B, a, S$ and $l$ are given by the following expressions:

- $\forall v_{1}, v_{2}, v_{3} \in V, B\left(v_{1}, v_{2}, v_{3}\right)=\left(f\left(v_{1}\right) . g\left(\nabla v_{2}\right), v_{3}\right)_{X}$, where $f$ and $g$ are respectively an $\mathbb{R}^{d}$ - valued Lipschitz application defined on $\mathbb{R}^{d}$ and an $\mathbb{R}^{d^{2}}-$ valued Lipschitz application defined on $\mathbb{R}^{d^{2}}$.

- $\forall v_{1}, v_{2} \in V, a\left(v_{1}, v_{2} ; \lambda\right)$ is a bilinear symmetric and positive form, which is continuous on $V \times V$ and coercive on $\left[H_{0}^{1}(\Omega)\right]^{d} \times\left[H_{0}^{1}(\Omega)\right]^{d}$. Moreover, we suppose that $a$ is $\alpha$-Holder with respect to $\lambda$, with Lipschitz constant equal to 1 , i.e., $\left|a\left(v_{1}, v_{2} ; \lambda_{1}\right)-a\left(v_{1}, v_{2} ; \lambda_{2}\right)\right| \leq\left\|\lambda_{1}-\lambda_{2}\right\|^{\alpha} a\left(v_{1}, v_{2}\right)$.

- $\forall v_{1}, v_{2} \in V, S\left(v_{1}, v_{2}\right)=\left(s\left(v_{1}\right), v_{2}\right)_{X}$, where $s$ is an $\mathbb{R}^{d}$-valued Lipschitz application defined on $\mathbb{R}^{d}$.

- $\forall v \in V, l_{t}(v)=(h(t), v)_{X}$, where $h$ is given in $L_{l o c}^{2}([0,+\infty[, X)$.

(.) denotes the scalar product in the corresponding space. And, $\lambda \in \mathbb{R}^{p,+, *}\left(p \in \mathbb{N}^{*}\right)$, denote the parameter vector of these equations.

A solution $u_{\lambda_{0}}$ of this equation associated with a reference parameter vector $\lambda_{0}$ is computed once and for all. A POD basis $\Phi^{\lambda_{0}}=\left(\Phi_{n}^{\lambda_{0}}\right)_{n>1}$ in $X$, associated with $u_{\lambda_{0}}$ on a time interval $(0, T)$, will lead to construct a ROM-POD describing the evolution of an approximation $\hat{u}_{\lambda, \lambda_{0}}$ of $u_{\lambda}$, in a subspace of dimension $N$ very small. We denote $\left(\mu_{n}^{\lambda_{0}}\right)_{n \geq 1}$ the POD eigenvalues sequence associated with the POD basis.

We know that:

$$
\frac{1}{T} \int_{0}^{T}\left(u_{\lambda_{0}}(t), \Phi_{n}^{\lambda_{0}}\right)_{X}^{2} d t=\mu_{n}^{\lambda_{0}}
$$

The problem that appears naturally, is to find a way to control the parametric evolution: what is $\frac{1}{T} \int_{0}^{T}\left(u_{\lambda}(t), \Phi_{n}^{\lambda_{0}}\right)_{X}^{2} d t$ ?

More precisely, if we denote $\forall t \in(0, T)$ and $\forall x \in X$ :

$$
\hat{u}_{\lambda, \lambda_{0}}(t, x)=\sum_{n=1}^{N} a_{n}^{\lambda, \lambda_{0}}(t) \Phi_{n}^{\lambda_{0}}(x),
$$


where $\forall n=1, \ldots, N, a_{n}^{\lambda, \lambda_{0}}(t)$ is the solution of:

$$
\left\{\begin{array}{l}
\frac{d a_{n}^{\lambda, \lambda_{0}}}{d t}+B\left(\hat{u}_{\lambda, \lambda_{0}}(t), \hat{u}_{\lambda, \lambda_{0}}(t), \Phi_{n}^{\lambda_{0}}\right)_{X}+a\left(\hat{u}_{\lambda, \lambda_{0}}(t), \Phi_{n}^{\lambda_{0}} ; \lambda\right) \\
=S\left(\hat{u}_{\lambda, \lambda_{0}}(t), \Phi_{n}^{\lambda_{0}}\right)+l_{t}\left(\Phi_{n}^{\lambda_{0}}\right) \\
a_{n}^{\lambda, \lambda_{0}}(0)=\left(u_{\lambda}^{0}, \Phi_{n}^{\lambda_{0}}\right)
\end{array}\right.
$$

Then, the question which arises naturally is the following: to what extent $\hat{u}_{\lambda, \lambda_{0}}$ remains an accurate approximation to $u_{\lambda}$ ?

\section{Main results}

In whats follows, we present our main results formally, with a lack in rigor:

\section{Reference POD basis associated with a reference parameter vector $\lambda_{0}$}

Formal result 1. There exist two decreasing sequences $\left(f_{1}^{\lambda_{0}}(N)\right)_{N \geq 1}$ and $\left(f_{2}^{\lambda_{0}}(N)\right)_{N \geq 1}$ ' such that:

$$
\left\|u_{\lambda}-\hat{u}_{\lambda, \lambda_{0}}\right\|_{L^{2}(0, T ; X)}^{2} \leq f_{1}^{\lambda_{0}}(N)+f_{2}^{\lambda_{0}}(N) \frac{\left\|\lambda-\lambda_{0}\right\|^{\alpha}}{\left\|\lambda_{0}\right\|}
$$

$f_{1}^{\lambda_{0}}(N)$ is the error induced already by taking $\lambda=\lambda_{0}$ :

$f_{1}^{\lambda_{0}}(N)=\left\|u_{\lambda_{0}}-\hat{u}_{\lambda_{0}}\right\|_{L^{2}(0, T ; X)}^{2}$, where $\hat{u}_{\lambda_{0}}=\hat{u}_{\lambda, \lambda_{0}}$, for $\lambda=\lambda_{0}$. For further details concerning the estimate of this term, we refer for instance to [38].

$f_{2}^{\lambda_{0}}(N)$ is a decreasing sequence, having the same rate of decline as the sequence $\left(\frac{1}{N}\right)_{N \geq 1}$

\section{Precision on result 1}

Heuristic result 1. Under regularity conditions on the solutions difference $u_{\lambda}-u_{\lambda_{0}} \in$ $L^{2}\left(0, T ;\left[H^{m}(\Omega)\right]^{d}\right)$ :

$$
\left\|u_{\lambda}-\hat{u}_{\lambda, \lambda_{0}}\right\|_{L^{2}(0, T ; X)}^{2} \leq f_{1}^{\lambda_{0}}(N)+c(N) \frac{\left\|\lambda-\lambda_{0}\right\|^{\alpha}}{\left\|\lambda_{0}\right\|},
$$

where $(c(N))_{N \geq 1}$ is a decreasing sequence, having the same rate of decline as the sequence $\left(\frac{1}{N^{m}}\right)_{N \geq 1}$

Improvement of the ROM-POD confidence interval: Enriched POD basis containing the parametric sensitivity of the solution

Formal result 2. Under the following restrictive conditions:

- $\nabla_{\lambda} u_{\lambda}\left(\lambda_{0}\right) \in L^{2}(0, T ; X)$.

- $\Phi^{\lambda_{0}}$ a POD basis associated to snapshots of $u_{\lambda_{0}}(t)$ and $\nabla_{\lambda} u_{\lambda}\left(\lambda_{0}\right)(t)$ on $(0, T)$.

We can show that:

$$
\left\|u_{\lambda}-\hat{u}_{\lambda, \lambda_{0}}\right\|_{L^{2}(0, T ; X)}^{2} \leq f_{1}^{\lambda_{0}}(N)+\sum_{n=N+1}^{\infty} \mu_{n}^{\lambda_{0}}\left\|\lambda-\lambda_{0}\right\|^{2}+K\left\|\lambda-\lambda_{0}\right\|^{4}
$$


Result 1 establishes an a priori estimate of the decrease rate of the squared ROM-POD error, especially when the two parameters $\lambda$ and $\lambda_{0}$ are distant.

Nevertheless, the usefulness of this result is improved thanks to result 1 under regularity conditions on the diffrenece between two solution flows associated respectively with a parameter $\lambda$ and the base parameter $\lambda_{0}$. Indeed, result 1 shows for a given class of solutions to equation (1), a very good efficiency of a reference reduced order model by a POD basis associated with a base parameter value $\lambda_{0}$.

Also, not to mention supplementary regularity conditions on the solution flows, the estimate (5) improves the validity domain of the reduced order model beside our previous result (estimate (3)) thanks to the term depending on $\left\|\lambda-\lambda_{0}\right\|^{4}$, and to the remainder of the expanded-POD eigenvalues sum multiplying $\left\|\lambda-\lambda_{0}\right\|^{2}$, of which decrease rate is optimal thanks to the POD construction.

\section{Organization of the paper}

In what follows, we give the proof elements of our principal result. In section "Results and discussion", we prove our formal results 1 and 2 and the heuristic result 1, in the context of multidimensional parametric problems. In section "Numerical experiments: The 2D Navier-Stokes equations", we present numerical experiments of our theoretical estimate for the 2D Navier-Stokes in the case of an unsteady and incompressible fluid flow. In section "Conclusion and prospects", we conclude by giving some prospects to this work.

\section{Results and discussion}

\section{Theorems and notations}

We recall some theorems and we define some notations, that could be useful to our proof.

\section{Theorem 1. Sobolev embeddings}

- For $d=1, H^{1}(\Omega) \subset L^{\infty}(\Omega)$, and $\exists C \in \mathbb{R}^{+*}$ such that $\forall v \in H^{1}(\Omega)$, $\|v\|_{L^{\infty}(\Omega)} \leq C\|\nabla v\|_{L^{2}(\Omega)}$.

- For $d=2, H^{1}(\Omega) \subset L^{4}(\Omega)$, and $\exists C \in \mathbb{R}^{+*}$ such that $\forall v \in H^{1}(\Omega)$, $\|v\|_{L^{4}(\Omega)} \leq C\|\nabla v\|_{\left[L^{2}(\Omega)\right]^{2}}$.

For more informations about Sobolev embeddings, see for instance [40].

\section{Theorem 2. Gagliardo-Nirenberg inequality}

- For $d=1, \exists c \in \mathbb{R}^{+*}$ such that $\|v\|_{L^{\infty}(\Omega)} \leq c\|v\|_{L^{2}(\Omega)}^{1 / 2}\|\nabla v\|_{L^{2}(\Omega)}^{1 / 2}, \forall v \in H^{1}(\Omega)$.

- For $d=2, \exists c \in \mathbb{R}^{+*}\|v\|_{L^{4}(\Omega)} \leq c\|v\|_{L^{2}(\Omega)}^{1 / 2}\|\nabla v\|_{\left[L^{2}(\Omega)\right]^{2}}^{1 / 2}, \forall v \in H^{1}(\Omega)$.

For more informations, see for instance [41].

Notation 1. $C_{p}^{\lambda}$ denotes the constant relative to the coercivity of the bilinear form $a$, associated with a given $\lambda$. So, $\forall v \in\left[H_{0}^{1}(\Omega)\right]^{d}$ :

$$
a(v, v ; \lambda) \geq C_{p}^{\lambda}\|\nabla v\|_{X}^{2}
$$


Notation 2. $K_{a}$ denotes the constant relative to the continuity of the bilinear form a on the spave V. So, $\forall v_{1}, v_{2} \in V$ :

$$
a\left(v_{1}, v_{2}\right) \leq K_{a}\left\|\nabla v_{1}\right\|_{X}\left\|\nabla v_{2}\right\|_{X} .
$$

Notation 3. $K_{f}, K_{g}$ and $K_{s}$ denote respectively the Lipschitz constants of the mappings $f$, $g$ and $s$.

Control of $\left\|u_{\lambda}-u_{\lambda_{0}}\right\|_{L^{2}(0, T ; X)}^{2}$

We recall the following intermediate lemma, based on the resolution of a Ricatti equation of order 3:

Lemma 1. Let a and b be two strictly positive real numbers, and let $z$ be a time dependent positive quantity verifying the following differential inequality:

$$
\frac{d z}{d t} \leq a z(t)+b z^{3}(t)
$$

Then,

$$
z(t) \leq\left(z^{-2}(0)+\frac{b}{a}(\exp (-2 a t)-1)\right)^{-\frac{1}{2}} .
$$

Proof. The proof of this lemma is essentially based on the following key points:

- $\quad$ The change of variable: $y(t)=z^{-2}(t)$.

- $\frac{d y}{d t} \geq-2 a y(t)-2 b$

- Application of the Gronwall lemma to inequality (6).

Thanks to lemma 1, we prove the following proposition:

Proposition 1. We show the following a priori upper bound of $\left\|u_{\lambda}-u_{\lambda_{0}}\right\|_{L^{2}(0, T ; X)}^{2}$, thanks to a differential Ricatti inequality in the later:

$$
\left\|\left(u_{\lambda}-u_{\lambda_{0}}\right)(t)\right\|_{X}^{2} \leq\left(\left\|u_{\lambda}^{0}-u_{\lambda_{0}}^{0}\right\|_{X}^{-4}+\frac{b}{a}(\exp (-2 a t)-1)\right)^{-\frac{1}{2}} K_{a}^{2}\left\|\nabla u_{\lambda_{0}}(t)\right\|_{X}^{2}\left\|\lambda-\lambda_{0}\right\|^{\alpha},
$$

where $a$ and $b$ are positive real numbers that will be detailed in what follows:

Proof. We prove this proposition for $d=1$. The same proof applies directly to the case: $d=2$.

We denote $w(t)=\left(u_{\lambda}-u_{\lambda_{0}}\right)(t)$, which verifies the following weak formulation:

$$
\left\{\begin{array}{l}
\frac{d}{d t}(w(t), v)_{X}+B\left(u_{\lambda}(t), u_{\lambda}(t), v\right)-B\left(u_{\lambda}(t), u_{\lambda_{0}}(t), v\right)+B\left(u_{\lambda}(t), u_{\lambda_{0}}(t), v\right) \\
-B\left(u_{\lambda_{0}}(t), u_{\lambda_{0}}(t), v\right)+a\left(u_{\lambda}(t), v ; \lambda\right)-a\left(u_{\lambda_{0}}(t), v ; \lambda_{0}\right) \\
=S\left(u_{\lambda}(t), v\right)-S\left(u_{\lambda_{0}}(t), v\right) \quad \forall v \in V \\
(w(0), v)_{X}=\left(u_{\lambda}^{0}-u_{\lambda}^{0}, v\right)_{X} \quad \forall v \in V
\end{array}\right.
$$


If we replace $v$ by $w(t)$, then we obtain:

$$
\begin{array}{r}
\frac{1}{2} \frac{d}{d t}\|w(t)\|_{X}^{2}+\int_{\Omega} f\left(u_{\lambda}(t)\right)\left(g\left(\nabla u_{\lambda}(t)\right)-g\left(\nabla u_{\lambda_{0}}(t)\right)\right) w(t) d x= \\
-\int_{\Omega}\left(f\left(u_{\lambda}(t)\right)-f\left(u_{\lambda_{0}}(t)\right)\right) g\left(\nabla u_{\lambda_{0}}(t)\right) w(t) d x-a(w(t), w(t) ; \lambda) \\
-a\left(u_{\lambda_{0}}(t), w(t) ; \lambda\right)+a\left(u_{\lambda_{0}}(t), w(t) ; \lambda_{0}\right)+\int_{\Omega}\left(s\left(u_{\lambda}(t)\right)-s\left(u_{\lambda_{0}}(t)\right)\right) w(t) d x .
\end{array}
$$

Therefore, by using the Sobolev embeddings mentioned above, we get:

$$
\begin{aligned}
\frac{1}{2} \frac{d}{d t}\|w(t)\|_{X}^{2} \leq & \left\|f\left(u_{\lambda}(t)\right)\right\|_{L^{\infty}(\Omega)}\left\|g\left(\nabla u_{\lambda}(t)\right)-g\left(\nabla u_{\lambda_{0}}(t)\right)\right\|_{X}\|w(t)\|_{X} \\
& +\left\|f\left(u_{\lambda}(t)\right)-f\left(u_{\lambda_{0}}(t)\right)\right\|_{L^{\infty}(\Omega)}\left\|g\left(\nabla u_{\lambda_{0}}(t)\right)\right\|_{X}\|w(t)\|_{X} \\
& -a(w(t), w(t) ; \lambda)-a\left(u_{\lambda_{0}}(t), w(t) ; \lambda\right)+a\left(u_{\lambda_{0}}(t), w(t) ; \lambda_{0}\right) \\
& +\left\|s\left(u_{\lambda}(t)\right)-s\left(u_{\lambda_{0}}(t)\right)\right\|_{X}\|w(t)\|_{X}
\end{aligned}
$$

Therefore,

$$
\begin{aligned}
\frac{1}{2} \frac{d}{d t}\|w(t)\|_{X}^{2} \leq & C K_{g}\left\|f\left(u_{\lambda_{0}}(t)\right)\right\|_{V}\|\nabla w(t)\|_{X}\|w(t)\|_{X} \\
& +K_{f} K_{g}\|w(t)\|_{L^{\infty}(\Omega)}\|\nabla w(t)\|_{X}\|w(t)\|_{X} \\
& +C K_{f}\left\|g\left(\nabla u_{\lambda_{0}}(t)\right)\right\|_{X}\|\nabla w(t)\|_{X}\|w(t)\|_{X} \\
& -C_{p}^{\lambda}\|\nabla w(t)\|_{X}^{2} \\
& +K_{a}\left\|\lambda-\lambda_{0}\right\|^{\alpha}\left\|\nabla u_{\lambda_{0}}(t)\right\|_{X}\|\nabla w(t)\|_{X}+K_{s}\|w(t)\|_{X}^{2}
\end{aligned}
$$

By appyling a Young inequality two times and the Gagliardo-Nirenberg inequality one time, we get:

$$
\begin{aligned}
\frac{1 d}{2} \frac{d}{d t}\|w(t)\|_{X}^{2} \leq & \left(\frac{C^{2}\left\|f\left(u_{\lambda_{0}}\right)\right\|_{V}^{2} K_{g}^{2}+C^{2}\left\|g\left(\nabla u_{\lambda_{0}}\right)\right\|_{X}^{2} K_{f}^{2}}{2 \beta}\right)\|w(t)\|_{X}^{2} \\
& +\beta\|\nabla w(t)\|_{X}^{2} \\
& +c K_{f} K_{g}\|w(t)\|_{X}^{3 / 2}\|\nabla w(t)\|_{X}^{3 / 2} \\
& -C_{p}^{\lambda}\|\nabla w(t)\|_{X}^{2} \\
& +\frac{1}{2 \varepsilon_{2}}\left\|\lambda-\lambda_{0}\right\|^{\alpha} K_{a}^{2}\left\|\nabla u_{\lambda_{0}}(t)\right\|_{X}^{2}+\frac{\varepsilon_{2}}{2}\left\|\lambda-\lambda_{0}\right\|^{\alpha}\|\nabla w(t)\|_{X}^{2} \\
& +K_{s}\|w(t)\|_{X}^{2}
\end{aligned}
$$

But $c K_{f} K_{g}\|w(t)\|_{X}^{3 / 2}\|\nabla w(t)\|_{X}^{3 / 2}$ can be upper bounded as follows, thanks to the application of a Young inequality two times:

$$
c K_{f} K_{g}\|w(t)\|_{X}^{3 / 2}\|\nabla w(t)\|_{X}^{3 / 2} \leq\|w(t)\|_{X}\left(\frac{c^{2} K_{f}^{2} K_{g}^{2} \delta}{2}\|w(t)\|_{X}+\frac{1}{2 \delta}\|\nabla w(t)\|_{X}^{3}\right)
$$


If we choose $\delta=\frac{\|w(t)\|_{X}\|\nabla w(t)\|_{X}}{\varepsilon_{3}}$, and $\eta=\frac{\|w(t)\|_{X}^{2}}{\varepsilon_{1}}$, then we obtain the following:

$$
\begin{aligned}
c K_{f} K_{g}\|w(t)\|_{X}^{3 / 2}\|\nabla w(t)\|_{X}^{3 / 2} \leq & \frac{\varepsilon_{3}}{2}\|\nabla w(t)\|_{X}^{2}+\frac{c^{2} K_{f}^{2} K_{g}^{2} \delta}{2}\|w(t)\|_{X}^{2} \\
\leq & \frac{\varepsilon_{3}}{2}\|\nabla w(t)\|_{X}^{2}+\frac{\delta^{2}}{8 \eta}+\frac{\eta c^{4} K_{f}^{4} K_{g}^{4}}{2}\|w(t)\|_{X}^{4} \\
\leq & \frac{\varepsilon_{3}}{2}\|\nabla w(t)\|_{X}^{2}+\frac{\varepsilon_{1}}{8\left(\varepsilon_{3}\right)^{2}}\|\nabla w(t)\|_{X}^{2} \\
& +\frac{c^{4} K_{f}^{4} K_{g}^{4}}{2 \varepsilon_{1}}\|w(t)\|_{X}^{6}
\end{aligned}
$$

Therefore,

$$
\begin{aligned}
\frac{1 d}{2} \frac{d}{d t}\|w(t)\|_{X}^{2} \leq & \frac{c^{4} K_{f}^{4} K_{g}^{4}}{2 \varepsilon_{1}}\|w(t)\|_{X}^{6} \\
& +\left(\frac{C^{2}\left\|f\left(u_{\lambda_{0}}\right)\right\|_{V}^{2} K_{g}^{2}+C^{2}\left\|g\left(\nabla u_{\lambda_{0}}\right)\right\|_{X}^{2} K_{f}^{2}}{2 \beta}+K_{s}\right)\|w(t)\|_{X}^{2} \\
& +\left(\beta+\frac{\varepsilon_{2}}{2}\left\|\lambda-\lambda_{0}\right\|^{\alpha}+\frac{\varepsilon_{3}}{2}+\frac{\varepsilon_{1}}{8\left(\varepsilon_{3}\right)^{2}}-C_{p}^{\lambda}\right)\|\nabla w(t)\|_{X}^{2} \\
& +\frac{1}{2 \varepsilon_{2}}\left\|\lambda-\lambda_{0}\right\|^{\alpha} K_{a}^{2}\left\|\nabla u_{\lambda_{0}}(t)\right\|_{X}^{2}
\end{aligned}
$$

If we choose $\beta, \varepsilon_{1}, \varepsilon_{2}$ and $\varepsilon_{3}$ such that the term multiplying $\|\nabla w(t)\|_{X}^{2}$ is negative, then by analogy to lemma 1 and by taking:

$$
a=2\left(\frac{C^{2}\left\|f\left(u_{\lambda_{0}}\right)\right\|_{V}^{2} K_{g}^{2}+C^{2}\left\|g\left(\nabla u_{\lambda_{0}}\right)\right\|_{X}^{2} K_{f}^{2}}{2 \beta}+K_{s}\right) .
$$

And,

$$
b=\frac{c^{4} K_{f}^{4} K_{g}^{4}}{\varepsilon_{1}}
$$

we conclude to our result.

\section{Remark 1. On the choice of $\beta, \varepsilon_{1}, \varepsilon_{2}$ and $\varepsilon_{3}$}

Let's suppose for instance, that $p=1, C_{p}^{\lambda}=\lambda C_{p}$ and $\alpha=1$.

A necessary condition to have $\beta<\lambda C_{p}-\frac{\varepsilon_{2}}{2}\left|\lambda-\lambda_{0}\right|-\frac{\varepsilon_{3}}{2}-\frac{\varepsilon_{1}}{8\left(\varepsilon_{3}\right)^{2}}$, is :

$$
\lambda C_{p}-\frac{\varepsilon_{2}}{2}\left|\lambda-\lambda_{0}\right|-\frac{\varepsilon_{3}}{2}-\frac{\varepsilon_{1}}{8\left(\varepsilon_{3}\right)^{2}}>0 .
$$

- If $\lambda>\lambda_{0}$, then equation (8) is equivalent to: $\left(C_{p}-\frac{\varepsilon_{2}}{2}\right) \lambda+\frac{\varepsilon_{2}}{2} \lambda_{0}-\frac{\varepsilon_{3}}{2}-\frac{\varepsilon_{1}}{8\left(\varepsilon_{3}\right)^{2}}>0$.

Then, we choose $\varepsilon_{1}, \varepsilon_{2}$ and $\varepsilon_{3}$ such that: $C_{p}>\frac{\varepsilon_{2}}{2}$, and $\frac{\varepsilon_{2}}{2} \lambda_{0}-\frac{\varepsilon_{3}}{2}-\frac{\varepsilon_{1}}{8\left(\varepsilon_{3}\right)^{2}}>0$. 
- If $\lambda<\lambda_{0}$, then equation (8) is equivalent to: $\left(C_{p}+\frac{\varepsilon_{2}}{2}\right) \lambda-\frac{\varepsilon_{2}}{2} \lambda_{0}-\frac{\varepsilon_{3}}{2}-\frac{\varepsilon_{1}}{8\left(\varepsilon_{3}\right)^{2}}>0$.

$$
\text { Then, } \lambda>\frac{\frac{\varepsilon_{2}}{2} \lambda_{0}+\frac{\varepsilon_{3}}{2}+\frac{\varepsilon_{1}}{8\left(\varepsilon_{3}\right)^{2}}}{C_{p}+\frac{\varepsilon_{2}}{2}} \text {. }
$$

\section{Choice of $\left(f_{2}^{\lambda_{0}}(N)\right)_{N \geq 1}$ and an a priori estimate of its terms}

We prove the following proposition, that will be a key point in order to give an a priori estimate of the terms of the sequence $\left(f_{2}^{\lambda_{0}}(N)\right)_{N=1, \ldots, M}$.

For instance, we suppose that $d=2$ and for convenience we take $\Omega=(0,1) \times(0,1)$, without any loss of generality:

Proposition 2. Let $\left(\Phi_{n}\right)_{n \geq 1}$ be an orthonormal basis of $\left(V,\|\cdot\|_{X}\right)$. We denote $\Phi_{n}=$ $\left(\Phi_{n}^{1}, \Phi_{n}^{2}\right)^{T}$. We define $f_{n}=\left(f_{n}^{1}, f_{n}^{2}\right)^{T}$ :

$f_{n}^{1}\left(x_{1}, y_{1}\right)=\int_{0}^{y_{1}} \int_{0}^{x_{1}} \Phi_{n}^{1}(x, y) d x d y \quad$ and $f_{n}^{2}\left(x_{1}, y_{1}\right)=\int_{0}^{y_{1}} \int_{0}^{x_{1}} \Phi_{n}^{2}(x, y) d x d y$.

Then, $\sum_{n=1}^{\infty}\left\|f_{n}\right\|_{X}^{2}=\frac{1}{2}$.

Proof. For $i=1,2$ :

$f_{n}^{i}(x, y)=\left(\Phi_{n}^{i}, 1_{[0, x] \times[0, y]}\right)_{X}$, then $\left\|f_{n}^{i}\right\|_{X}^{2}=\int_{0}^{1} \int_{0}^{1}\left|\left(\Phi_{n}^{i}, \mathbf{1}_{[0, x] \times[0, y]}\right)_{X}\right|^{2} d x d y$.

Therefore, $\sum_{n=1}^{\infty}\left\|f_{n}^{i}\right\|_{X}^{2}=\int_{0}^{1} \int_{0}^{1}\left\|\mathbf{1}_{[0, x] \times[0, y]}\right\|_{X}^{2} d x d y$.

Which concludes to the result.

From now on, we denote: $f_{2}^{\lambda_{0}}(N)=\sum_{n=N+1}^{\infty}\left\|f_{n}^{\lambda_{0}}\right\|_{X}^{2}$, where $f_{n}^{\lambda_{0}}$ is the primitive function of the POD mode $\Phi_{n}^{\lambda_{0}}$.

\section{Completion of the proof of result 1}

$$
\begin{aligned}
\left\|u_{\lambda}-\hat{u}_{\lambda, \lambda_{0}}\right\|_{L^{2}(0, T ; X)}^{2} \leq & 2\left\|u_{\lambda_{0}}-\hat{u}_{\lambda_{0}}\right\|_{L^{2}(0, T ; X)}^{2} \\
& +2\left\|\left(u_{\lambda}-u_{\lambda_{0}}\right)-\Pi_{\Phi^{\lambda_{0}}}^{N}\left(u_{\lambda}-u_{\lambda_{0}}\right)\right\|_{L^{2}(0, T ; X)}^{2} \\
& +2\left\|\Pi_{\Phi^{\lambda_{0}}}^{N}\left(u_{\lambda}-u_{\lambda_{0}}\right)-\left(\hat{u}_{\lambda, \lambda_{0}}-\hat{u}_{\lambda_{0}}\right)\right\|_{L^{2}(0, T ; X)}^{2}
\end{aligned}
$$

- $\left\|\left(u_{\lambda}-u_{\lambda_{0}}\right)-\Pi_{\Phi^{\lambda_{0}}}^{N}\left(u_{\lambda}-u_{\lambda_{0}}\right)\right\|_{L^{2}(0, T ; X)}^{2}=\sum_{n=N+1}^{\infty}\left\|\left(u_{\lambda}-u_{\lambda_{0}}, \Phi_{n}^{\lambda_{0}}\right)_{X}\right\|_{L^{2}(0, T ; \mathbb{R})}^{2}$.

- The Galerkin error $\left\|\Pi_{\Phi^{\lambda_{0}}}^{N}\left(u_{\lambda}-u_{\lambda_{0}}\right)-\left(\hat{u}_{\lambda, \lambda_{0}}-\hat{u}_{\lambda_{0}}\right)\right\|_{L^{2}(0, T ; X)}^{2}$ is controled by $\sum_{n=N+1}^{\infty}\left\|\left(u_{\lambda}-u_{\lambda_{0}}, \Phi_{n}^{\lambda_{0}}\right)_{X}\right\|_{L^{2}(0, T ; \mathbb{R})}^{2} ;$ This is shown easily by reducing the equation describing the evolution of $\left(u_{\lambda}-u_{\lambda_{0}}\right)(t)$. 
Therefore, the parametric squared POD-Galerkin error is essentially controled by the remainder $\sum_{n=N+1}^{\infty}\left\|\left(u_{\lambda}^{h}-u_{\lambda_{0}}^{h}, \Phi_{n}^{\lambda_{0}}\right)_{X}\right\|_{L^{2}(0, T ; \mathbb{R})}^{2}$. Then, based on the previous proposition 2, a way to study the decrease rate of this remainder will be by considering the remainder of the primitives sum of the reference POD modes $\Phi_{n}^{\lambda_{0}}$ : This is shown simply by applying successively the Green formula and the Cauchy-Schwarz inequality to each one of the orthogonal projection coefficients $\left(\left(u_{\lambda}-u_{\lambda_{0}}\right)(t), \Phi_{n}^{\lambda_{0}}\right)_{X}$.

Therefore,

$$
\sum_{n=N+1}^{\infty}\left\|\left(u_{\lambda}-u_{\lambda_{0}}, \Phi_{n}^{\lambda_{0}}\right)_{X}\right\|_{L^{2}(0, T ; \mathbb{R})}^{2} \text { is controled by } f_{2}^{\lambda_{0}}(N)\left\|\lambda-\lambda_{0}\right\|^{\alpha} .
$$

This ends the proof of result 1 .

\section{Completion of the proof of heuristic result 1}

By applying successively the Green formula and the Cauchy-Schwarz inequality and by repeating this step m-times to each $\left(\left(u_{\lambda}-u_{\lambda_{0}}\right)(t), \Phi_{n}^{\lambda_{0}}\right)_{X}$, we prove that $\sum_{n=N+1}^{\infty}\left\|\left(u_{\lambda}-u_{\lambda_{0}}, \Phi_{n}^{\lambda_{0}}\right)_{X}\right\|_{L^{2}(0, T ; \mathbb{R})}^{2}$ is finally controled by the remainder of the m-iterated primitives sum of the reference POD modes.

Which concludes to the result.

\section{Completion of the proof of result 2}

Thanks to proposition (1), the first restrictive condition of result 2 is verified for a quasinonlinear equation of the form (1). Then, we write the following Taylor expansion of $u_{\lambda}$ to the order 1:

$u_{\lambda}=u_{\lambda_{0}}+\nabla_{\lambda} u_{\lambda}\left(\lambda_{0}\right) .\left(\lambda-\lambda_{0}\right)+R_{1}(\lambda)$, where $\left\|R_{1}(\lambda)\right\|_{L^{2}(0, T ; X)}$ is a function of $\left\|\lambda-\lambda_{0}\right\|^{2}$.

Now, we impose the second restrictive condition of result 2 . Then, we can easily show that:

$$
\left\|\left(\nabla_{\lambda} u_{\lambda}\left(\lambda_{0}\right), \Phi_{n}^{\lambda_{0}}\right)_{X}\right\|_{L^{2}(0, T ; \mathbb{R})}^{2} \leq 2 T \mu_{n}^{\lambda_{0}} .
$$

In this case, the remainder $\sum_{n=N+1}^{\infty}\left\|\left(u_{\lambda}-u_{\lambda_{0}}, \Phi_{n}^{\lambda_{0}}\right)_{X}\right\|_{L^{2}(0, T ; \mathbb{R})}^{2}$ is better controled by the POD modes:

$$
\begin{aligned}
\sum_{n=N+1}^{M}\left\|\left(u_{\lambda}-u_{\lambda_{0}}, \Phi_{n}^{\lambda_{0}}\right)_{X}\right\|_{L^{2}(0, T ; \mathbb{R})}^{2} \leq & \left\|\lambda-\lambda_{0}\right\|^{2} \sum_{n=N+1}^{\infty}\left\|\left(\nabla_{\lambda} u_{\lambda}\left(\lambda_{0}\right), \Phi_{n}^{\lambda_{0}}\right)_{X}\right\|_{L^{2}(0, T ; \mathbb{R})}^{2} \\
& +\left\|R_{1}(\lambda)\right\|_{L^{2}(0, T ; X)}^{2}
\end{aligned}
$$

Therefore, we deduce the a priori estimate (5).

This ends the proof.

\section{Numerical experiments: The 2D Navier-Stokes equations}

We place our problem in the particular case of the Navier-Stokes equations for a 2D incompressible fluid flow. The parameter $\lambda$ denotes here the viscosity of the flow. 


\section{Flow configuration}

Our study configuration is for an unsteady and incompressible fluid flow in a channel, around a circular cylinder (see Figure 1). The inlet condition is a uniform fluid flow. We impose an outlet condition, and symmetry conditions on $\Gamma_{1}$ and $\Gamma_{2}$.

\section{The two strategies of POD computations}

We present a numerical comparaison of our theoretical estimates (3) and (5), for a fixed POD modes numbers $N$ and by varying only the viscosity $\lambda$. For a fixed POD modes number $N$, we plot the logarithm of parametric ROM-POD error $\left\|u_{\lambda}^{h}-\hat{u}_{\lambda, \lambda_{0}}\right\|_{L^{2}(0, T ; X)}$, as a function of $\log \left(\left|\lambda-\lambda_{0}\right|\right)$.

Indeed, the reference parameter value $\lambda_{0}=0.001$ corresponds to a Reynolds number equal to 100 . And, we varied the viscosity $\lambda$ in the interval $\left[5.56 \times 10^{-4}, 1.43 \times 10^{-3}\right]$ in order to have computations associated with Reynolds numbers varying in the interval $[70,180]$. We consider two strategies of POD computations. Indeed, two different snapshots sets are considered on the time interval $[0, T]$, where $T=75 \mathrm{~s}$ :

- $\mathcal{S}_{1}=\left\{u_{\lambda_{0}}(t) \quad t \in[0, T]\right\}$.

- $\mathcal{S}_{2}=\left\{v_{\lambda_{0}}(t) t \in[0,2 T]\right\}$. Such that:

$$
\begin{aligned}
& -v_{\lambda_{0}}(t)=u_{\lambda_{0}}(t) \text { for } t \in[0, T] . \\
& -v_{\lambda_{0}}(t)=\frac{\partial u}{\partial \lambda}\left(\lambda_{0}\right)(2 T-t) \text { for } t>T .
\end{aligned}
$$

More precisely, if we discretize the time interval to $M=200$ points, then the snapshots sets are given as follows:

- $\mathcal{S}_{1}=\left\{u_{\lambda_{0}}\left(t_{i}\right) \quad i=1, \ldots, M\right\}$.

- $\mathcal{S}_{2}=\left\{v_{\lambda_{0}}\left(t_{i}\right)\right.$ for $\left.i=1, \cdots, 2 M\right\}$

Therefore, the POD eigenvectors $\Phi_{n}^{\lambda_{0}}$ associated respectively with these snapshots sets are solutions of the following two eigenvalues problems:

The correlation matrix:

$$
C_{i j}^{1}=\int_{\Omega} u_{\lambda_{0}}\left(t_{i}, x, y\right) \cdot u_{\lambda_{0}}\left(t_{j}, x, y\right) d \Omega
$$

of which size is $M \times M$.

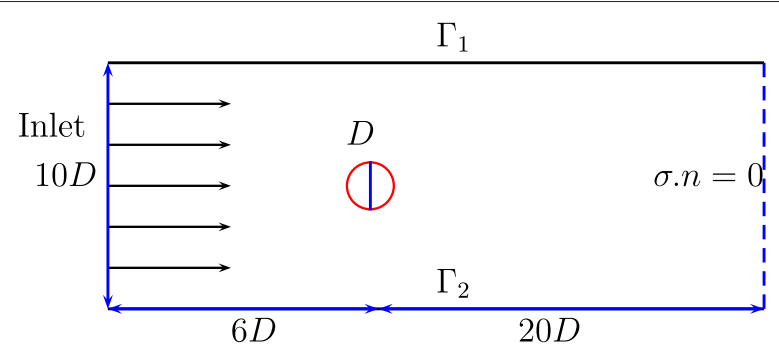

Figure 1 Flow configuration. 
We denote by $A_{n}=\left(A_{i, n}\right)_{1 \leq i \leq M}$ for $n=1, \ldots, M$, a set of orthonormal eigenvectors of the matrix $C^{1}$. Then, the POD-eigenvectors associated to $u_{\lambda_{0}}$, are given by:

$$
\Phi_{n}^{\lambda_{0}}=\frac{1}{\sqrt{M}} \sum_{i=1}^{M} A_{i, n} u_{\lambda_{0}}\left(t_{i}\right)
$$

And, the correlation matrix:

$$
C_{i j}^{2}=\int_{\Omega} v_{\lambda_{0}}\left(t_{i}, x\right) \cdot v_{\lambda_{0}}\left(t_{j}, x\right) d \Omega,
$$

of which size is $2 M \times 2 M$.

We denote by $A_{n}=\left(A_{i, n}\right)_{1 \leq i \leq 2 M}$ for $n=1, \ldots, 2 M$, a set of orthonormal eigenvectors of the matrix $C^{2}$. Then, the POD-eigenvectors associated to $v_{\lambda_{0}}$, are given by:

$$
\Phi_{n}^{\lambda_{0}}=\frac{1}{\sqrt{2 M}} \sum_{i=1}^{2 M} A_{i, n} \nu_{\lambda_{0}}\left(t_{i}\right) .
$$

We should note that, in the case of $\mathcal{S}_{2}$, the dimensions of the snapshots are compatible. Indeed, the flow snapshots are normalized by the inlet velocity, and the parametric derivatives snapshots are normalized by their corresponding magnitudes.

\section{Numerical comparaison of the two theoretical estimates (3) and (5)}

We compare the two plots of $\log \left(\left\|u_{\lambda}-\hat{u}_{\lambda, \lambda_{0}}\right\|_{L^{2}(0, T ; X)}\right)$ as a function of $\log \left(\left|\lambda-\lambda_{0}\right|\right)$, obtained respectively from the model reduction by the reference POD basis and the enriched one. We get the two plots on (Figure 2).

We retrieve here the power law of the parametric ROM-POD error with respect to $\left|\lambda-\lambda_{0}\right|$, for both strategies of the POD computation.

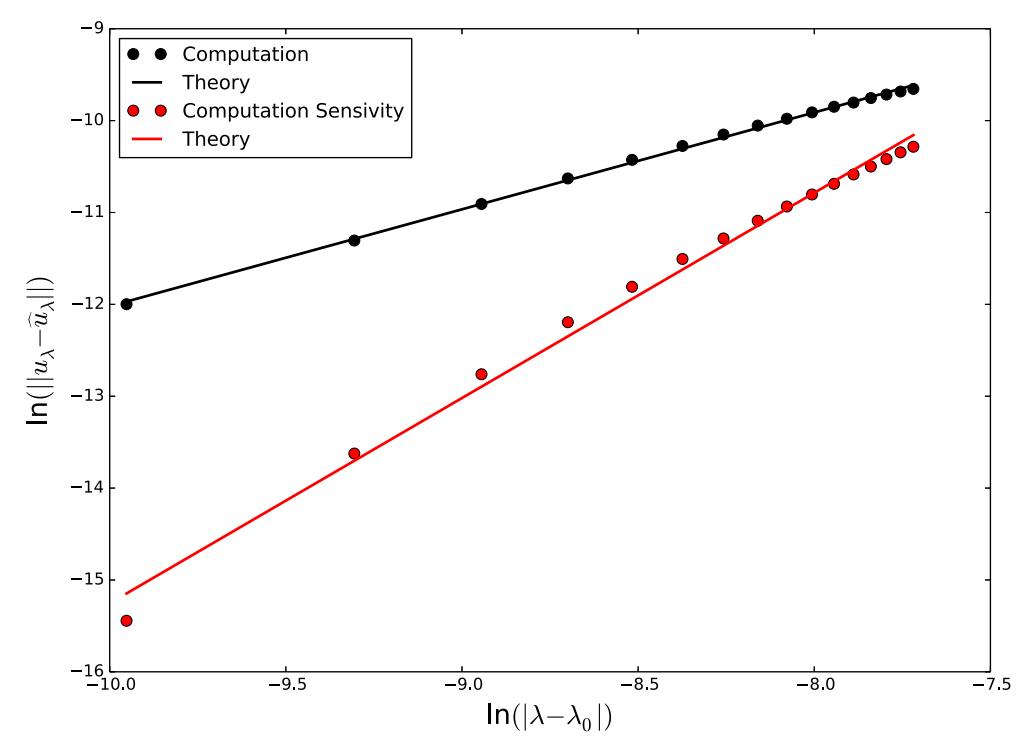

Figure 2 Red line: The logarithm of the error relative to the model reduction by the enriched POD basis. Black line: The logarithm of the error relative to the model reduction by the reference POD basis. 
Moreover, (Figure 2) shows that the case for which the parametric sensitivity of the flow is also included in the snapshots set, is better than the one where we had only snapshots associated with a reference solution: The slope of the red line is clearly greater than the black one, and the numerical parametric error in this case is less than the one associated to the reduction by a reference POD basis.

\section{Conclusion and prospects \\ Conclusion}

We were interested in mathematical a priori error bounds of a parametric ROM by a reference POD. A mathematical a priori estimate of the parametric squared $L^{2}$-error induced by the corresponding ROM is developped, thanks to the resolution of a Ricatti differential inequality.

This result is an improvement of the one we developed in [38], where we showed an a priori estimate of the parametric squared $L^{2}$-error induced after the model reduction of parameterized semi-discrete quasi-linear parabolic problems, by a reference POD basis associated with a reference solution. We obtained here sharper a priori estimates. Moreover, when we considered the enriched POD basis, the parametric ROM-POD error was better controled. Which shows an improvement of the ROM-POD confidence interval with respect to parametric variation.

\section{Prospects}

Concerning the prospects of this work, we hope to be able to apply this type of results as an a priori convergence technique of enrichement algorithms as the POD-Greedy one.

It is important also to be able to use these a priori techniques for errors estimate as a sharp a posteriori errors indicator.

Furthermore, it is important to apply these results to interpolation techniques (using the Lie group theory) of reduced order models, available each one in a confidence region around a reference parameter.

Competing interests

The authors declare that they have no competing interests.

\section{Authors' contributions}

NA have made substantive intellectual contributions to the mathematical conception of the manuscript and drafted the manuscript. AH have been involved in revising the manuscript critically for important intellectual content and have given final approval of the version to be published. EL have made substantial contributions to the acquisition of the numerical data and to the interpretation of the numerical experiments. MJ have participated to the mathematical content of the manuscript. All authors read and approved the final manuscript.

Acknowledgment

The authors are thankful to FEDER (European Regional Development Fund) for providing the necessary financial facilities for the preparation of the paper.

Received: 23 December 2013 Accepted: 7 July 2014

Published: 2 August 2014

\section{References}

1. Lieu T, Farhat C, Lesoinne M (2006) Reduced-order fluid/structure modeling of a complete aircraft configuration. Comput Methods Appl Mech Engrg 195(41-43):5730-5742

2. Balajewicz M, Farhat C (2013) Model order reduction of embedded boundary models. Bulletin of the American Physical Society 58 
3. Liberge E, Hamdouni A (2010) Reducer order modeling method via proper orthogonal decomposition (POD) for flow around an oscillating cylinder. J Fluids Struct 26(2):292-311

4. Liberge E, Benaouicha M, Hamdouni A (2007) Proper Orthogonal Decomposition (POD) investigation in fluid stucture interaction. Eur J Comput Mech 16:401-418

5. Liberge $E$, Benaouicha M, Hamdouni A (2008) Low order dynamical system for fluid rigid body interaction problem using POD method. Int J Multiphys 2:59-81

6. Liberge $E$, Pomarède $M$, Hamdouni A (2010) Reduced-order modeling by POD multiphase approach for fluid-structure interaction. Eur J Comput Mech 19:41-52

7. Lassila T, Quarteroni A, Rozza G (2012) A reduced basis model with parametric coupling for fluid-structure interaction problems. SIAM J Sci Comput 34(2):1187-1213

8. Lassila T, Manzoni A, Rozza G (2012) On the approximation of stability factors for general parametrized partial differential equations with a two-level affine decomposition. ESAIM Math Model Numer Anal 46(6):1555-1576

9. Manzoni A, Quarteroni A, Rozza G (2012) Shape optimization for viscous flows by reduced basis methods and free-form deformation. Int J Numer Methods Fluids 70(5):646-670

10. Lassila T, Rozza G (2010) Parametric free-form shape design with pde models and reduced basis method. Comput Methods Appl Mech Eng 199(23-24):1583-1592

11. Ito K, Ravindran SS (1998) A reduced-order method for simulation and control of fluid flows. J Comp Phys 143:403-425

12. Afanasiev K, Hinze M (2001) Adaptive control of a wake flow using proper orthogonal decomposition. Shape optimization optimal design. Lecture Notes Pure Appl Math:317-332

13. Maday Y, Patera AT, Turinici G (2002) Global a priori convergence theory for reduced-basis approximations of single-parameter symmetric coercive elliptic partial differential equations. C R Acad Sci Paris, Ser I 335:289-294

14. Buffa A, Maday Y, Patera AT, Prud'homme C, Turinici G (2012) A priori convergence theory of the greedy algorithm for the parametrized reduced basis. ESAIM-Math Model Numer Anal 46(3):595-603

15. Chen Y, Hesthaven J-S, Maday Y, Rodriguez J, Zhu X (2012) Certified reduced basis method for electromagnetic scattering and radar cross section estimation. Comput Methods Appl Mech Eng 92(108):233-236

16. Machiels L, Maday Y, Patera AT (2001) Output bounds for reduced-order approximations of elliptic partial differential equations. Comput Methods Appl Mech Eng 190:3413-3426

17. Grepl MA, Maday Y, Nguyen N-C, Patera AT (2007) Efficient reduced-basis treatment of nonaffine and nonlinear partial differential equations. ESAIM: Math Model Numer Anal 41(3):575-605

18. Klindworth D, Grepl MA, Vossen G (2012) Certified reduced basis methods for parametrized parabolic partial differential equations with non-affine source terms. Comput Methods Appl Mech Eng 209(212):144-155

19. Veroy K, Prud'homme C, Patera AT (2003) Reduced-basis approximation of the viscous burgers equation: rigorous a posteriori error bounds. C R Acad Sci Paris, Ser I 337:619-624

20. Nguyen N-C, Rozza G, Patera AT (2009) Reduced basis approximation and a posteriori error estimation for the time-dependent viscous Burgers' equation. Calcolo 46:157-185

21. Ladeveze P, Passieux J-C, Néron D (2010) The LATIN multiscale computational method and the proper generalized decomposition. Comput Methods Appl Mech Eng 199(21-22):1287-1296

22. Ladeveze P, Nouy A (2003) On a multiscale computational strategy with time and space homogenization for structural mechanics. Comput Methods Appl Mech Eng 192(28-30):3061-3087

23. Ammar A, Chinesta F, Diez P, Huerta A (2010) An error estimate for seperated representation of highly multidimensional models. Comput Methods Appl Mech Eng 199:1872-1880

24. Ammar A, Mokdad B, Chinesta F, Keunings R (2006) A new family of solvers for some classes of multidimensionnal partial differential equations encountered in kinetic theory modeling of complex fluids. J Non Newtonian Fluid Mech 139:153-176

25. Ammar A, Normandin M, Chinesta F (2010) Solving parametric complex fluids models in rheometric fows. J Non Newtonian Fluid Mech 165(23-24):1588-1601

26. Chinesta F, Ammar A, Cueto E (2010) Recent advances and new challenges in the use of the proper generalized decomposition for solving multidimensional models. Arch Comput Methods Eng 17(4):327-350

27. Chinesta F, Leygue A, Bordeu F, Aguado JV, Cueto E, Gonzalez D, Alfaro I, Ammar A, Huerta A (2013) Pgd-based computational vademecum for efficient design, optimization and control. Arch Comput Methods Eng 20(1):31-59. doi:10.1007/s11831-013-9080-x

28. Gonzalez D, Masson F, Poulhaon F, Leygue A, Cueto E, Chinesta F (2012) Proper generalized decomposition based dynamic data driven inverse identification. Math Comput Simul 82(9):1677-1695. doi:10.1016/j.matcom.2012.04.001

29. Berkooz G, Holmes P, Lumley JL (1993) The proper orthogonal decomposition in the analysis of turbulent flows. Annu Rev Fluid Mech 25(1):539-575

30. Aubry N, Holmes P, Lumley JL, Stone E (1988) The dynamics of coherent structures in the wall region of a turbulent boundary layer. J Fluid Mech 192:115-173

31. Amsallem D, Cortial J, Carlberg K, Farhat C (2009) A method for interpolation on manifolds structural dynamics reduced-order models. Int J Numer Methods Eng 80:1241-1258

32. Amsallem D, Farhat C (2008) An interpolation method for adapting reduced order models and application to aeroelasticity. Am Inst Aeronautic Astronautics 46(7):1803-1813

33. Amsallem D, Cortial J, Farhat C (2009) On-demand CFD-based aeroelastic predictions using a database of reduced-order bases and models In: 47th AIAA Aerospace Sciences Meeting, vol 18.

34. Hinze M, Volkwein S (2008) Error estimates for abstract linear-quadratic optimal control problems using proper orthogonal decomposition. Comput Optim Appl 39:319-345

35. Troltzsch F, Volkwein S (2009) POD a-posteriori error estimates for linear-quadratic optimal control problems. Comput Optim Appl 44:83-115

36. Tonn T, Urban K, Volkwein S (2011) Comparison of the reduced-basis and POD a posteriori error estimators for an elliptic linear-quadratic optimal control problem. Math Comput Model Dyn Syst 17(4):355-369 
37. Vossen G, Volkwein S (2012) Model reduction techniques with a posteriori error analysis for linear quadratic optimal control problems. Math Comput Model Dyn Syst 2(3):465-485

38. Akkari N, Hamdouni A, Liberge E, Jazar M (2013) A mathematical and numerical study of the sensitivity of a reduced order model by POD ROM-POD, for a 2D incompressible fluid flow. J Comput Appl Math 270:522-530

39. Akkari N, Hamdouni A, Jazar M Mathematical and numerical results on the parametric sensitivity of a ROM-POD of the burgers equation. Eur J Comput Mech 23(1-2):78-95

40. Evans L (1998) Partial differential equations. Am Math Soc 662

41. Nirenberg L (1959) On elliptic partial differential equations. Ann Scuola Norm Sup Pisa 13(3):115-162

doi:10.1186/s40323-014-0014-4

Cite this article as: Akkari et al: On the sensitivity of the POD technique for a parameterized quasi-nonlinear parabolic equation. Advanced Modeling and Simulation in Engineering Sciences 2014 2:14

\section{Submit your manuscript to a SpringerOpen ${ }^{\circ}$} journal and benefit from:

- Convenient online submission

- Rigorous peer review

- Immediate publication on acceptance

- Open access: articles freely available online

- High visibility within the field

- Retaining the copyright to your article

Submit your next manuscript at $\gg$ springeropen.com 